\title{
Metformin With Neoadjuvant Chemoradiation to Improve Pathologic Response in Rectal Cancer: a Pilot Phase I/II Trial
}

Shun Wong ( $\nabla$ shun.wong@sunnybrook.ca )

University of Toronto https://orcid.org/0000-0001-6292-1188

William Chu

Sunnybrook Health Sciences Centre

Shady Ashamalla

Sunnybrook Health Sciences Centre

Darlene Fenech

Sunnybrook Health Sciences Centre

Scott Berry

Queen's University

Alex Kiss

Institute for Clinical Evaluative Sciences

Marianne Koritzinsky

University of Toronto Faculty of Medicine

Research article

Keywords: rectal cancer, metformin, neoadjuvant chemoradiation

Posted Date: August 4th, 2020

DOI: https://doi.org/10.21203/rs.3.rs-48488/v1

License: (c) (i) This work is licensed under a Creative Commons Attribution 4.0 International License. Read Full License

Version of Record: A version of this preprint was published at Clinical and Translational Radiation Oncology on July 1st, 2021. See the published version at https://doi.org/10.1016/j.ctro.2021.07.001. 


\section{Abstract}

Background: Neoadjuvant radiotherapy with or without chemotherapy decreases the risk of local recurrence after surgery for stage II or III rectal cancer. Emerging data suggest that diabetic patients on metformin may have improved cancer outcome after radiotherapy. We asked if metformin given concurrently with long course chemoradiation (CRT) may improve pathologic complete response (pCR) in non-diabetic rectal cancer patients. A single-institutional pilot study was performed to build a confidence interval for the pCR rate and to determine the sample size for a phase 2 trial.

Methods: Non-diabetic patients with biopsy confirmed adenocarcinoma of the rectum, and deemed candidates for long course neoadjuvant CRT were invited to participate. Radiation consisted of 50.4 Gy in 28 daily fractions. Capecitabine ( $825 \mathrm{mg} / \mathrm{m}^{2}$ twice daily, Monday-Friday) was self-administered during the 28 days of radiation only. The primary outcome was pCR. The study was designed to accrue 15 participants to construct a confidence interval (Cl) for the $\mathrm{pCR}$ rate.

Results: A total of 16 patients were accrued from January 2017 to May 2018. One patient withdrew from the study prior to CRT. Only grade 1 or 2 adverse events were observed from the intervention. Three patients had a clinical complete response (cCR) and did not have surgical resection. Of the 12 patients who underwent surgery, there were two pCRs. For the combined pCR/cCR rate of $33 \%$ (95\% $\mathrm{Cl} 19-47 \%)$, a total of 85 patients will be required to yield a $95 \% \mathrm{Cl}$ with a $10 \%$ margin of error.

Conclusions: Adding metformin to neoadjuvant CRT for rectal cancer does not appear to enhance toxicities. These results will be used to refine the design and conduct of a future phase 2 trial to determine whether adding metformin to CRT improves pCR/cCR rates.

Trial registration: NCT03053544. Registered December 20, 2016, https://clinicaltrials.gov/ct2/show/record/NCT03053544

\section{Article Summary}

\section{Strengths and limitations of this study}

- This was the first prospective trial to evaluate the role of concurrent metformin with chemoradiation as neoadjuvant treatment for rectal cancer.

- It was a pilot study designed to construct a confidence interval for the pathologic complete response rate.

- Although the primary endpoint was complete pathological response rate, a few participants elected not to undergo surgery following a clinical complete response.

- There might be potential for bias on outcome due to sex/gender with only a single woman in the cohort.

- None of the participants consented to repeat biopsies for the translational component of the study.

\section{Background}

Pre-operative or neoadjuvant radiotherapy with or without chemotherapy decreases the risk of local recurrence after surgery for rectal cancer, and is currently considered standard therapy for patients with stage II or III rectal cancer [1]. For patients with locally advanced rectal cancer, optimizing the neoadjuvant therapy may further improve locoregional control. Neoadjuvant chemoradiation (CRT) results in $15-25 \%$ pathological complete response (pCR) rates. Patients with a pCR may not benefit from definitive surgical resection $[2,3]$. Thus, improving CR rates has the potential to also spare a significant number of patients from the morbidity of surgery including the need for a permanent stoma or low anterior resection syndrome.

Over the last decade, great interest has emerged in the potential use of metformin, a biguanide commonly used as first line of treatment for type 2 diabetics, in cancer treatment [4]. A growing body of epidemiologic data has demonstrated that metformin reduces cancer incidence and mortality including colorectal cancer[5-7]. In a retrospective study, diabetic rectal cancer patients on metformin was found to have improved pCR rates after neoadjuvant CRT [8]. A few recent trials reported that metformin decreased tumor cell proliferation in non-diabetic patients [9-11]. Metformin also suppressed the number and size of premalignant colorectal aberrant crypt foci [12]. These clinical data have been corroborated by pre-clinical studies where metformin was shown to reduce xenograft growth after radiotherapy [13]. Prospective trials are thus warranted to assess the potential benefits of metformin in non-diabetic patients with rectal cancer.

We hypothesize that metformin improves rates of $\mathrm{PCR}$ in patients with rectal cancer treated with neoadjuvant CRT. The objective was to evaluate the feasibility of using metformin to improve pCR rates in non-diabetic patients undergoing standard of care neoadjuvant CRT for rectal cancer. The primary end-point was the $\mathrm{pCR}$ rate and its confidence interval $(\mathrm{Cl})$ to allow us to determine the sample size for a larger phase 2 trial.

\section{Methods}

\section{Study overview}

This pilot phase I/II study (ClinicalTrials.gov: NCT03053544) was conducted in compliance with the trial protocol, applicable institutional Research Ethics Board (REB, \#472-2015) requirements and policies, International Conference on Harmonization Good Clinical Practice (IHC-GCP) E6 guidelines and Part C Division 5 (Drugs for Clinical Trials Involving Human Subjects) of the Food and Drug Regulations as per Health Canada (No Objection Letter, metformin control number 194654). See online supplementary file 1 for the protocol of the study. The REB approved Informed Consent Form (ICF) was obtained according to ICH-GCP guidelines and institutional REB policy. The complete original ICF (supplementary file 2) was maintained by the site, with a copy 
provided to the participant. Site monitoring was conducted by the Institutional Clinical Trials Services to ensure the safety of human study participants and compliance of study processes and documentation based on approved protocols and regulatory policies.

This study was conducted in a large tertiary cancer centre in Toronto, Ontario, Canada.

\section{Participants}

All participants who met all of the following inclusion criteria were identified and were invited to participate in this study:

1. Male or female aged 19 or older.

2. Plan for care inclusive of:

1. standard of care neoadjuvant CRT

2. planned total mesorectal excision (TME)

3. Histologically confirmed adenocarcinoma of the rectum.

4. Completed staging investigations including magnetic resonance imaging (MRI) of pelvis, computerized tomography (CT) of chest/abdomen/pelvis, complete blood counts (CBC), liver function test including aspartate aminotransferase (AST) or alanine aminotransferase (ALT) and bilirubin, creatinine, carcinoembryonic antigen.

5. At least one of the following:

1. T3 or T4 lesion

2. $\mathrm{T} 2$ lesion $\leq 1 \mathrm{~mm}$ to the mesorectal fascia

3. Node positive rectal tumor

6. ECOG performance status of 0 or 1 .

Patients with a diagnosis of diabetes, life expectancy of 6 months or less, prior pelvic radiation, serum creatinine $>1.5 \times$, AST or ALT $>2.5 \times$ or bilirubin $>1.5 \times$ upper limit of normal were ineligible. Pregnant or breastfeeding women were also excluded from participation in this study.

\section{Intervention}

Following informed consent (supplementary file 2), participants were assigned a unique study identifier and dispensed the study drug, metformin. The intervention consisted of 3 phases.

Phase 1: 2 weeks prior to CRT

Participants self-administered $500 \mathrm{mg}$ of metformin twice daily by mouth starting 1 to 2 weeks prior to the start of CRT.

Phase 2: during CRT

Participants continued metformin daily throughout the course of CRT. Radiation consisted of 50.4 Gy in 28 daily fractions. All patients underwent CT simulation and radiation was delivered by volumetric intensity-modulated arc therapy with daily cone beam CT for image guidance. Capecitabine, $825 \mathrm{mg} / \mathrm{m}^{2}$ twice daily was self-administered during the 28 days of radiation only. Participants were seen weekly during CRT including one study visit during CRT scheduled $4 \pm 1$ week after the start of CRT. This visit included review of metformin compliance, a blood test to for CBC, creatinine, AST/ALT and bilirubin, adverse events and updates of concomitant medications.

Phase 3: post-CRT

Participants continued metformin at a dose of $500 \mathrm{mg}$ twice daily, with the last dose at 4 weeks $\pm \llbracket 3$ days after completion of CRT. A study visit was scheduled $7 \pm 3$ days after the stop date of metformin to coincide with the end of the adverse event reporting time period. At this visit, unused metformin tablets and participant pill diaries were collected, metformin compliance reviewed, adverse events documented and assessed and changes to concomitant medications recorded. All participants were evaluated for adverse events from the time of their first treatment with metformin. Adverse events were graded based on the Common Terminology Criteria for Adverse Events (CTCAE) Version 4. Surgery typically occurs at approximately 2 months after end of CRT as part of standard of care at the Institution.

\section{Statistical and sample size considerations}

All participants who received at least one week of CRT were considered completed participants and evaluable for response. The primary endpoint was the pCR rate. The definition of $\mathrm{PCR}$ was total regression, no tumor cells or fibrotic tissue only with or without mucin based on the surgical pathology report. The study was to be an internal pilot study to allow for construction of a confidence interval for the pCR rate. Since 12 participants have been shown to be the minimum number required to build a confidence interval [14], the study was designed to have a sample size of 15 completed participants. Participants who did not complete the study were to be replaced.

The pCR rate and its associated 95\% confidence intervals was estimated using unadjusted normal approximation for binomial proportions ( $z$ approximation). Upon completion of the internal pilot study, the data from all participants were used to derive a formal sample size calculation for a future phase 2 trial.

Page $3 / 8$ 
Patients were invited to participate in an optional translational study which involved a separate consent. This included a "research" biopsy by flexible sigmoidoscopy to be done after a minimum of one-week of metformin and prior to CRT. This translationl component was designed to assess tumor proliferation and hypoxia, and markers of metformin activity longitudinally using the tumor sample from the initial diagnostic biopsy, "research" biopsy taken at the start of CRT, and the final surgical specimen if applicable. Unfortunately none of the participants consented to having the optional sigmoidoscopy and biopsy. The translational component was thus not performed.

\section{Results}

A total of 16 patients (age 34-81) were accrued from Jan 2017-Dec 2018. There were 15 men and one woman. One patient developed grade 2 nausea and emesis after the first three doses of metformin and withdrew from the study. One patient underwent emergency surgery for an ischemic ileostomy and stopped metformin at $27 \mathrm{~Gy}$. This adverse event was deemed not an adverse drug reaction and unrelated to metformin based on the Safety Monitoring Plan. He went on to complete CRT without metformin after revision of the ileostomy. This patient was considered evaluable for response as defined in the protocol. The remaining 14 patients all completed CRT as per protocol. All participants indicated that they took metformin as per protocol. One patient did not return the metformin pill bottle to the institutional pharmacy. Only 9 of 15 participants returned their metformin diaries at the end of the intervention. Table 1 outlined the clinical characteristics of the 15 patients.

Table 1

Clinical characteristics and pathological responses

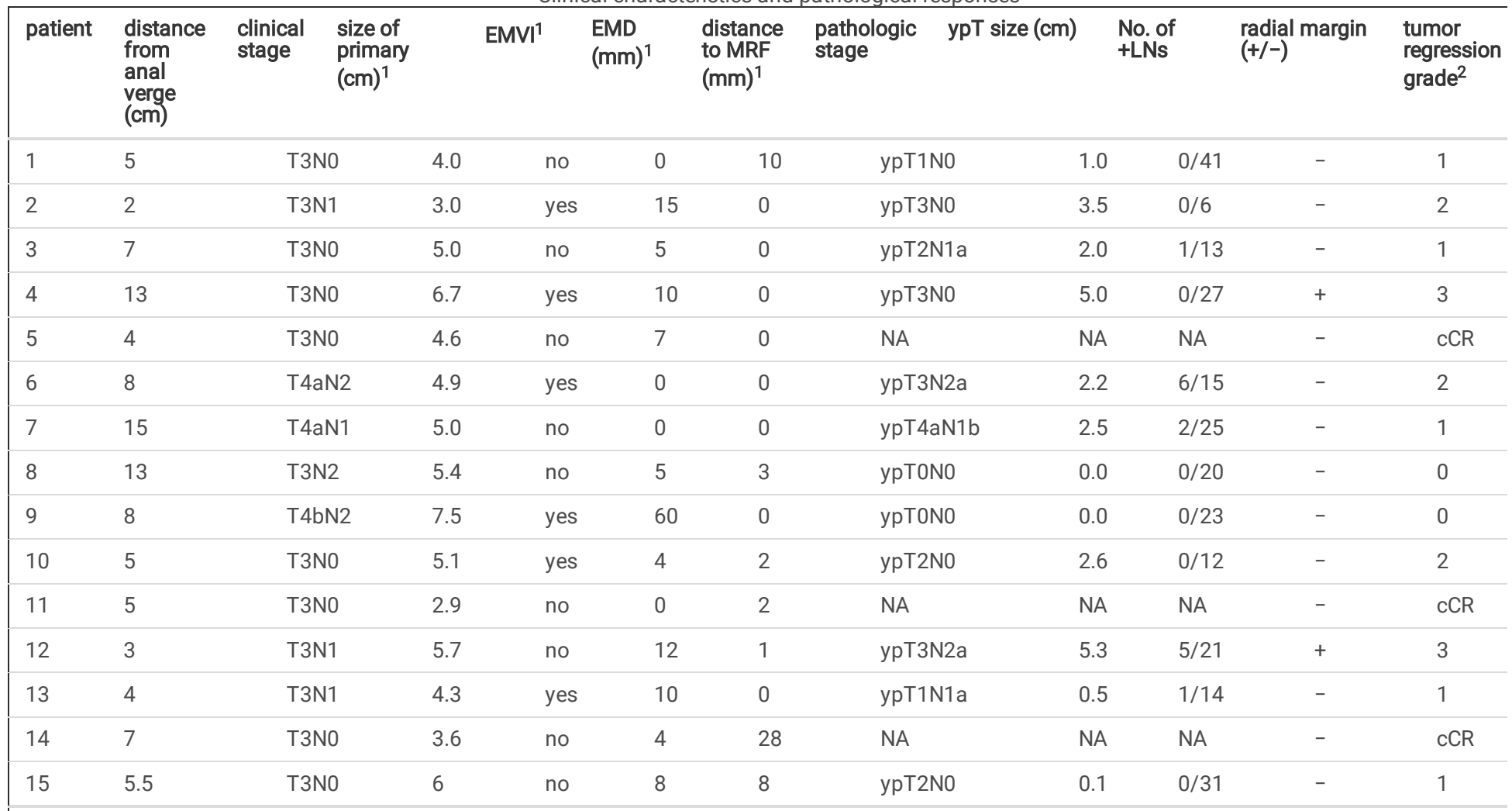

EMVI, extramural venous invasion; EMD, extramural depth; MRF, mesorectal fascia; +LNs, positive lymph nodes; +/-, positive/negative; cCR, clinical complete response; NA, not applicable

${ }^{1}$ based on MRI

${ }^{2}$ American Joint Committee on Cancer tumor regression grade: $0=$ pathologic complete response, $1=$ minimal residual disease, $2=$ minor response, $3=$ no response

With the exception of the patient who developed an ischemic stoma, only grade 1 or 2 adverse events were observed (Table 2). 
Table 2

Adverse event grades according to Common Terminology Criteria for Adverse Events, Version 4.02

\begin{tabular}{|c|c|c|c|c|c|c|c|c|c|}
\hline patient & nausea & vomiting & fatigue & hand-foot syndrome & proctitis & cystitis & diamhea & dermatitis & other \\
\hline 1 & - & - & - & - & - & - & - & - & - \\
\hline 2 & 1 & - & - & - & - & - & - & - & - \\
\hline 3 & - & - & - & 1 & 1 & - & - & - & - \\
\hline 4 & - & - & - & - & - & - & - & - & $4^{a}$ \\
\hline 5 & - & - & - & - & 1 & - & - & - & - \\
\hline 6 & 1 & 1 & - & - & - & - & - & - & $1^{\mathrm{b}}$ \\
\hline 7 & - & 2 & 1 & - & - & - & - & - & - \\
\hline 8 & 1 & - & - & 1 & 1 & 1 & 1 & 2 & - \\
\hline 9 & - & - & - & - & - & - & 1 & - & - \\
\hline 10 & - & - & - & - & 2 & 2 & - & 1 & - \\
\hline 11 & 1 & - & - & - & 1 & 1 & 1 & - & - \\
\hline 12 & 1 & 1 & 1 & - & - & 1 & - & - & - \\
\hline 13 & - & - & - & 1 & 1 & - & 1 & - & - \\
\hline 14 & 1 & - & 1 & - & - & - & 1 & - & - \\
\hline 15 & 1 & - & - & - & - & 1 & - & - & $1^{c}$ \\
\hline \multicolumn{10}{|c|}{ a small bowel obstruction from ischemia of ileostomy requiring surgery } \\
\hline \multicolumn{10}{|c|}{ bdizziness, myalgia, elevated creatinine } \\
\hline${ }^{c}$ myalgi & & & & & & & & & \\
\hline
\end{tabular}

Three patients had a clinical complete response (cCR) and declined to have surgical resection (Table 1). All 3 remained disease free at 25, 26 and 33 months from date of informed consent on our institutional surveillance protocol. Of the 12 patients who underwent surgery (abdominoperineal resection: 2 , low anterior resection: 10) between 2-3 months after CRT, there were two PCR. Five patients had minimal residual disease, 3 had minor response, and 2 had minimal response based on the American Joint Committee on Cancer tumor regression grades. For the combined pCR/CR rate of 33\% (95\% Cl 19-47\%), a sample size of 85 patients will be needed in the phase 2 study to have a $95 \% \mathrm{Cl}$ that the CR rate is within $10 \%$ of the observed value.

\section{Discussion}

Several randomized trials have shown that neoadjuvant radiotherapy with or without chemotherapy decreases the risks of local recurrence after surgery for rectal cancer [15-18]. Neoadjuvant therapy is currently considered standard therapy for patients with stage II or III rectal cancer. These treatments do not affect survival and are associated with poorer functional outcomes such as bowel and sexual function compared with surgery alone. Recent advances in surgical techniques such as complete mesorectal resection and pre-operative MRI have suggested that MRI criteria may select patients who can be managed with surgery alone [19]. Improving the accuracy of preoperative staging is an active area of research that allows for the selection of patients who will benefit from a neoadjuvant approach. Many patients with locally advanced rectal cancer have unresectable disease or continue to develop local recurrence despite neoadjuvant CRT and aggressive resection. Optimizing the tumor response and identifying potential biomarkers represent other strategies that may lead to improved outcome. Neoadjuvant CRT results in 15-20\% pCR rates. The majority of patients with a pCR may not benefit from definitive surgical resection [2, 3]. Improving the $\mathrm{pCR}$ rates has the potential to spare a significant number of patients from the morbidity of surgery such as the need for a permanent stoma or low anterior resection syndrome.

There is extensive preclinical data to suggest that metformin has shown that metformin inhibits cancer proliferation [5, 20]. Metformin is known to inhibit mitochondrial activity, leading to reduced respiration and altered redox states [21]. These biologic effects may represent the underlying mechanism of inhibition of cell proliferation by metformin $[20,22]$. In addition to inhibition of cancer cell proliferation, metformin might improve radiation response by decreasing tumor hypoxia-mediated radiation resistance [13]. There is preclinical evidence that metformin serves as a radiosensitizing agent and improves radiation treatment response in various cancer cell lines including colorectal cancer $[13,23]$. Metformin has thus been proposed as a novel radiation modifier $[22,24]$.

The optional translational study was designed with the goal to obtain a study "biopsy" to assess cancer cell proliferation and hypoxia 1-2 weeks after metformin and compared these biological parameters with those from the initial biopsies. Recruiting patients to participate in the translational arm of the study turned out to be challenging as none of the participants agreed to have a repeat sigmoidoscopy and biopsy outside of standard of care. Obtaining tissue samples serially for translational studies is important to better understand the putative mechanisms of experimental interventions. Strategies to 
enhance patients' willingness to undergo additional sigmoidoscopy and biopsy is needed. Pertinent translational or biologic information will also need to be derived from the original biopsies or surgical specimens.

When the study was designed, the primary endpoint was PCR. Three patients elected for non-operative management demonstrated no evidence of residual or recurrent cancer at follow-up at over 2 years. It is expected that had these patients undergone TME, they would have had a pCR. In studies on neoadjuvant treatment for rectal cancer, it is expected some patients with a cCR will be reluctant to undergo surgery. Indeed, cCR patients at our institution are typically offered the option of non-operative management and close surveillance. We thus reported a combined $\mathrm{pCR} / \mathrm{cCR}$ rate and considered this value appropriate for future trial design.

Surprisingly of the 16 patients who consented to participate, there was only a single woman. It is unclear why this was the case. In the screening log book for participants, there was no apparent difference in the number of men or women screened or deemed eligible. In a recent analysis of the National Cancer Database, odds of achieving pCR were independently associated with female sex. Hence, the observed pCR/CR rate of $33 \%$ was unlikely skewed due to the over-representation of male patients [25].

Metformin has been used for decades in patients with type 2 diabetes and has an extremely safe toxicity profile. With current interest in the use of metformin as a cancer therapeutic in non-diabetics, this study served to provide proof-of-principle data that metformin could be safely integrated into the neoadjuvant treatment of rectal cancer and to provide data to design an appropriately powered Phase 2 trial to further study this concept.

\section{Abbreviations}

CRT: chemoradiation; pCR: pathological complete response; Cl: confidence interval; REB: Research Ethics Board; IHC-GCP: International Conference on Harmonization Good Clinical Practice; ICF: Informed Consent Form; TME: total mesorectal excision; MRI: magnetic resonance imaging; CT: computerized tomography; CBC: complete blood counts; AST: aspartate aminotransferase; ALT: alanine aminotransferase; CTCAE: Common Terminology Criteria for Adverse Events; cCR: clinical complete response.

\section{Declarations}

\section{Acknowledgements}

The authors thank the Gastrointestinal Site Group and the Clinical Trials Services of the Odette Cancer Centre, Sunnybrook Health Sciences Centre for their review of the protocol and support of the trial.

\section{Author information}

Affiliations

Department of Radiation Oncology, Sunnybrook Health Sciences Centre, University of Toronto, Ontario, Canada

C. Shun Wong, William Chu

Division of Surgical Oncology, Department of Surgery, Sunnybrook Health Sciences Centre, University of Toronto, Ontario, Canada

Shady Ashamalla, Darlene Fenech

Department of Oncology, Queen's University, Ontario, Canada

Scott Berry

Institute for Clinical Evaluative Sciences, Institute of Health Policy, Management and Evaluation, University of Toronto, Ontario, Canada

Alex Kiss

Department of Radiation Oncology, Princess Margaret Cancer Centre, University of Toronto, Ontario, Canada

Marianne Koritzinsky

\section{Contributions}

CSW and MK contributed to the initial design of the study. CSW, WC, SA, DF, SB contributed to recruitment of participants and data collection. AK contributed to the statistical analysis. CSW drafted the first version of the article. All authors contributed to data analysis and interpretation, revised the article and approved the final version for submission for publication. CSW had final responsibility for the decision to submit for publication. CSW had overall responsibility for the research design and implementation, including data collection, analysis and write-up.

\section{Corresponding author}

Correspondence to C. Shun Wong

\section{Funding}


The work was supported by a peer-reviewed grant from the Department of Radiation Oncology, University of Toronto (UTDRO Collaborative Seed Grant 2014). The Funder of this study had no influence in the design of the study, the collection, analysis and interpretation of the data, or the writing of the manuscript. The corresponding author had full access to all the data in the study and had responsibility for the decision to submit for publication.

\section{Availability of data and materials}

All data relevant to the study are included in the article.

\section{Ethics approvaland consent to participate}

This study was approved by the Research Ethics Board (REB) of Sunnybrook Health Sciences Centre (REB \#472-2015). Prior to REB submission, the trial was reviewed and approved by the Gastrointestinal Site Group of the Odette Cancer Centre, Sunnybrook Health Sciences Centre. All participants provided signed informed consent (supplementary file 2).

\section{Consent for publication}

All participants provided consent for publication of results of the study.

\section{Competing interests}

The authors have no conflicts to declare pertaining to this study.

\section{References}

1. Kennedy E, Vella ET, Blair Macdonald D, Wong CS, McLeod R, Cancer Care Ontario Preoperative Assessment for Rectal Cancer Guideline Development G. Optimisation of preoperative assessment in patients diagnosed with rectal cancer. Clin Oncol. 2015; 27(4):225-245.

2. O'Neill BD, Brown G, Heald RJ, Cunningham D, Tait DM. Non-operative treatment after neoadjuvant chemoradiotherapy for rectal cancer. Lancet Oncol. 2007; 8(7):625-633.

3. Smith CA, Kachnic LA. Evolving treatment paradigm in the treatment of locally advanced rectal cancer. J Natl Compr Cancer Netw. $2018 ; 16(7)$ : 909-915.

4. Pollak M.The insulin and insulin-like growth factor receptor family in neoplasia: an update. Nature Rev Cancer. 2012, 12(3):159-169.

5. Evans JM, Donnelly LA, Emslie-Smith AM, Alessi DR, Morris AD. Metformin and reduced risk of cancer in diabetic patients. BMJ. 2005; 330(7503):13041305.

6. Noto H, Goto A, Tsujimoto T, Noda M. Cancer risk in diabetic patients treated with metformin: a systematic review and meta-analysis. PloS One. 2012; 7(3):e33411.

7. Mei ZB, Zhang ZJ, Liu CY, Liu Y, Cui A, Liang ZL, Wang GH, Cui L. Survival benefits of metformin for colorectal cancer patients with diabetes: a systematic review and meta-analysis. PloS One. 2014; 9(3):e91818.

8. Skinner HD, Crane CH, Garrett CR, Eng C, Chang GJ, Skibber JM, Rodriguez-Bigas MA, Kelly P, Sandulache VC, Delclos ME et al. Metformin use and improved response to therapy in rectal cancer. Cancer Med. 2013; 2(1):99-107.

9. Joshua AM, Zannella VE, Downes MR, Bowes B, Hersey K, Koritzinsky M, Schwab M, Hofmann U, Evans A, van der Kwast T et al. A pilot 'window of opportunity' neoadjuvant study of metformin in localised prostate cancer. Prostate Cancer Prostatic Dis. 2014;17(3):252-258.

10. Niraula S, Dowling RJ, Ennis M, Chang MC, Done SJ, Hood N, Escallon J, Leong WL, McCready DR, Reedijk M et al. Metformin in early breast cancer: a prospective window of opportunity neoadjuvant study. Breast Cancer Res Treat. 2012; 135(3):821-830.

11. Laskov I, Drudi L, Beauchamp MC, Yasmeen A, Ferenczy A, Pollak M, Gotlieb WH. Anti-diabetic doses of metformin decrease proliferation markers in tumors of patients with endometrial cancer. Gynecol Oncol. 2014; 134(3):607-614.

12. Hosono K, Endo H, Takahashi H, Sugiyama M, Sakai E, Uchiyama T, Suzuki K, lida H, Sakamoto Y, Yoneda K et al. Metformin suppresses colorectal aberrant crypt foci in a short-term clinical trial. Cancer Prev Res. 2010; 3(9):1077-1083.

13. Zannella VE, Dal Pra A, Muaddi H, McKee TD, Stapleton S, Sykes J, Glicksman R, Chaib S, Zamiara P, Milosevic M et al. Reprogramming metabolism with metformin improves tumor oxygenation and radiotherapy response. Clin Cancer Res. 2013; 19(24):6741-6750.

14. van Belle G. Statistical rules of thumb. Wiley-Interscience 2008.

15. Kapiteijn E, Marijnen CA, Nagtegaal ID, Putter H, Steup WH, Wiggers T, Rutten HJ, Pahlman L, Glimelius B, van Krieken JH et al. Preoperative radiotherapy combined with total mesorectal excision for resectable rectal cancer. N Engl J Med. 2001; 345(9):638-646.

16. Sauer R, Becker H, Hohenberger W, Rodel C, Wittekind C, Fietkau R, Martus P,Tschmelitsch J, Hager E, Hess CF et al. Preoperative versus postoperative chemoradiotherapy for rectal cancer. N Engl J Med. 2004; 351(17):1731-1740.

17. Bujko K, Nowacki MP, Nasierowska-Guttmejer A, Michalski W, Bebenek M, Kryj M. Long-term results of a randomized trial comparing preoperative shortcourse radiotherapy with preoperative conventionally fractionated chemoradiation for rectal cancer. Br J Surg. 2006; 93(10):1215-1223.

18. Sebag-Montefiore D, Stephens RJ, Steele R, Monson J, Grieve R, Khanna S, Quirke P, Couture J, de Metz C, Myint AS et al. Preoperative radiotherapy versus selective postoperative chemoradiotherapy in patients with rectal cancer (MRC CR07 and NCIC-CTG C016): a multicentre, randomised trial. Lancet 2009; 373(9666):811-820.

19. Kennedy ED, Simunovic M, Jhaveri K, Kirsch R, Brierley J, Drolet S, Brown C, Vos PM, Xiong W, MacLean T et al. Safety and Feasibility of Using Magnetic Resonance Imaging Criteria to Identify Patients With "Good Prognosis" Rectal Cancer Eligible for Primary Surgery: The phase 2 nonrandomized 
QuickSilver clinical trial. JAMA Oncol. 2019; 5(7):961-966.

20. Foretz M, Guigas B, Bertrand L, Pollak M, Viollet B. Metformin: from mechanisms of action to therapies. Cell Metab. 2014; 20(6):953-966.

21. Madiraju AK, Qiu Y, Perry RJ, Rahimi Y, Zhang XM, Zhang D, Camporez JG, Cline GW, Butrico GM, Kemp BE et al. Metformin inhibits gluconeogenesis via a redox-dependent mechanism in vivo. Nat Med. 2018; 24(9):1384-1394.

22. Koritzinsky M: Metformin: A novel biological modifier of tumor response to radiation therapy. Int J Radiat Oncol Biol Phys. 2015; 93(2):454-464.

23. Fernandes JM, Jandrey EHF, Koyama FC, Leite KRM, Camargo AA, Costa ET, Perez RO, Asprino PF. Metformin as an alternative radiosensitizing agent to 5fluorouracil during neoadjuvant treatment for rectal cancer. Dis Colon Rectum. 2020; 63(7): 918-926.

24. Samsuri NAB, Leech M, Marignol L: Metformin and improved treatment outcomes in radiation therapy - a review. Cancer Treat Rev. 2017 ; 55:150-162.

25. Lorimer PD, Motz BM, Kirks RC, Boselli DM, Walsh KK, Prabhu RS, Hill JS, Salo JC: Pathologic complete response rates after neoadjuvant treatment in rectal cancer: an analysis of the National Cancer Database. Ann Surg Oncol. 2017; 24(8):2095-2103.

\section{Supplementary Files}

This is a list of supplementary files associated with this preprint. Click to download.

- CONSORTPilotFeasibilityTrialsChecklistMetformin.docx

- BMCCancerSuppfile2ICF.pdf

- BMJCancerSuppfile1metforminCRT.pdf 